\title{
LA NOCIÓN DE LA CANTIDAD REPRESENTADA METAFÓRICAMENTE EN LOS PROVERBIOS ESPAÑOLES ${ }^{1}$
}

\author{
SVETLANA PIKUL
}

Universidad Estatal de Voronezh, Rusia

\begin{abstract}
Resumen
El objetivo de este trabajo es examinar la representación metarórica de la noción de la cantidad en los proverbios españoles. Como resultado del análisis realizado, se determinaron algunos modos de la representación metafórica de la cantidad. Una de las categorías de la existencia, la cantidad se expresa en el proverbio español a través de los conceptos básicos de la vida del pueblo: el tiempo, el espacio, la medida, la distancia, la forma y la unidad monetaria.
\end{abstract}

Palabras clave: cantidad, metáfora, proverbio, categoría, concepto.

\begin{abstract}
The aim of this paper is to examine the metaphoric representation of the notion of quantity in the Spanish proverbs. As a result of the realized analysis, some modes of the metaphorical representation of the quantity was determined. As one of the categories of existence, the quantity is expressed in the Spanish proverb through the basic concepts of the life of the people: time, space, measure, distance, form and the monetary unit.
\end{abstract}

Key words: quantity, metaphor, proverb, category, concept.

La lengua es la forma esencial de la representación del mundo objetivo. La lengua contiene y traspasa de generación en generación la experiencia acumulada, el conocimiento, el sistema de valores y todos los conceptos de un pueblo sobre el mundo y las leyes de la existencia. La lengua es capaz de abarcar con su léxico y semántica toda la cultura polifacética. «Por medio de los recursos del idioma uno puede fijar los conocimientos sobre los ambientes naturales y culturales en los que vive y actúa» (Grishaeva, 2008: 222).

Entre la gran variedad de los medios de la representación de la realidad objetiva, la más eficaz es la fraseología, ya que representa un papel especial en la transmisión de la conciencia cultural de una nación y su identificación, encarnando, en el sentido figurado de las unidades del idioma, su percepción del universo desde el punto de vista cultural (Teliya, 1996: 235).

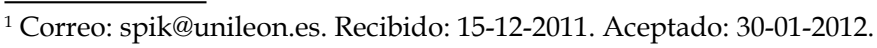


El objeto de la investigación del presente artículo es la representación metafórica de la noción de cantidad en los proverbios españoles. La cantidad es la categoría filosófica que expresa la cantidad, el número, el volumen, el grado del desarrollo de las características del objeto. Todo lo que es capaz de aumentar o disminuir y que puede medirse o numerarse. En la lengua, la categoría de la cantidad es una de las categorías clave, con un amplio espectro de formas de expresarla. En el habla popular la cantidad puede representar metafóricamente los objetos y los acontecimientos del mundo real.

El material de la investigación para el presente artículo no ha sido elegido casualmente. Los proverbios son «el código espiritual del pueblo» (Anikin, 1957: 10), reflejan el espíritu popular, las aspiraciones y los ideales, los juicios sobre diferentes aspectos de la vida. Los proverbios y refranes de cualquier lengua representan los productos de la conciencia de lengua común, la experiencia de las generaciones y de los representantes del pueblo. En este artículo examinamos los proverbios españoles que contienen la idea de la cantidad expresada metafóricamente.

«La metáfora atraviesa la vida cotidiana de la persona, estructurando la percepción, el pensamiento y la actividad, la metáfora está presente en el sistema conceptual de la persona» (Lakoff, Johnson). En el espacio científico-matemático, la idea de la cantidad se traspasa exactamente, es «mucho» / «poco», ola cantidad exacta de algo, o la aproximación (pero con la presencia invariable del lexema «aproximadamente»). Y sólo la metáfora da el número enorme de variantes de la expresión de la idea de la cantidad, lo que podemos ver examinando los proverbios españoles.

Como resultado del análisis realizado, se determinaron algunos modos de la representación metafórica de la cantidad. La cantidad puede ser expresada a través de la forma, la distancia, la medida, el tiempo, el espacio, la unidad monetaria, al igual que por medio de las estructuras sintácticas (la comparación y la contraposición). Se determinaron diez grupos de proverbios que contenían metáforas cuantitativas.

\section{PROVERBIOS QUE EXPRESAN LA IDEA DE LA CANTIDAD GRANDE O PEQUEÑA POR MEDIO DE LA COMPARACIÓN/CONTRAPOSICIÓN}

\subsection{La comparación de objetos con la forma grande/pequeña}

Dale un buen huevo al codicioso y te pedirá la gallina. (= pedirá mucho)

Dar una gallina por un huevo, no lo apruebo. (= dar mucho por poco)

Hombre celoso, hace de una pulga un oso. (= de poco - mucho)

\subsection{La comparación de una parte del objeto con el objeto entero}

No des ni un dedo al villano, que se tomará la mano. (= tomará mucho) 


\subsection{La comparación de un objeto con otro determinando la utilidad/valor del objeto}

Es necesario notar que el objeto con que se realiza la comparación casi siempre es de escaso valor, es decir el objeto/sujeto no vale nada. En el proverbio esta idea se expresa por medio de la comparación con un objeto muy pequeño (la semilla), con moneda menuda, con un objeto necesario de la ropa (pantalones), o con lo que se considera tradicionalmente innecesario/poco útil (el barro) y se usa a menudo para la comparación:

\footnotetext{
Hombre grandón, no vale un cañamón. (= poco o nada)

Mesa sin vino no vale un comino. (= poco o nada)

La gloria humana no vale una avellana. (= poco)

Salud y dinero haya, que lo demás no vale dos habas. (= poco o nada)

Ira sin fortaleza, no vale ni media cereza. (= poco)

Caldo sin jamón ni gallina, no vale una sardina. (= poco o nada)

Aceituna cordobí para boca toledana no vale un maravedí. (= poco)

Dinero encerrado, no gana un cornado. (= poco)

Caballo argel, no des ni un real por él. (= nada) (el real $=0,25$ pesetas)

Juntos veinte ladrones, no pueden robar a quien no lleva ni calzones. (= poco)

Guitarra sin cuerdas no vale una mierda. (= poco)

La presencia del dueño, vale tanto como el estiércol. (= poco)
}

Sin embargo, a veces la comparación tiene carácter positivo y, entonces, el objeto se compara con una gran cantidad de algo de valor:

Manos de dama no sirven para nada; manos de oficial valen un caudal. (= mucho)

\subsection{La comparación acerca de la cualidad de un objeto con otro}

Una hora de contento vale por ciento. (= mucho)

Una palabra del que sabe, dice más que el discurso de un ignorante. (= vale mucho)

Así, vemos que las figuras de la comparación y la contraposición tienen gran potencial para expresar la idea de la cantidad: grande o pequeña. Como material para la comparación sirven además las realidades de costumbre, más difundidas y propias del pueblo español. La mayoría de objetos de comparación son objetos domésticos propios del pueblo español.

\section{PROVERBIOS QUE DESIGNAN EL CONCEPTO DE LA CANTIDAD A TRAVÉS DE LA DISTANCIA}

Lo que el concepto de la cantidad se expresa a través de la distancia, indica el enlace fundamental entre la cantidad y el espacio. 


\subsection{La distancia expresada por la medida de la longitud}

En este caso, el numeral cardinal no expresa la cantidad exacta, sino la cantidad grande o pequeña:

De lo pintado a lo vivo, hay cien leguas de camino; y de lo vivo a lo pintado, otro tanto. (= mucho)

De prometer a dar, hay unas leguas de mal andar. (= mucho)

De Ubeza a Baea (Jaén), una legua. (= poco)

Hombres hay ruínes, aunque miden el oro por celemines. (= mucho) (celemín - la medida de los áridos $=4,625)$

De prometer a dar, hay unas leguas de mal andar. (= mucho)

Del loco al airado no va un palmo. (= poco)

De los albañiles y de la suegra, a cien leguas. (= mucho)

2.2 La distancia expresada por la medida aproximada, "pública" de la longitud, como el paso Entre el loco y el airado, media un paso. (= poco)

De santo a diablo, un paso; de diablo a santo un trecho largo. ( 1 = poco; 2 = mucho)

De rico a ruín hay un paso de cagachín. (= poco)

Hace falta decir que el lexema cagachín en este proverbio indica la distancia muy pequeña.

De mercader a ladrón, va un saltito de gorrión. (= poco)

De mercader a ladrón, un escalón. (= poco)

Aquí se expresa al mismo tiempo la idea de la distancia y del tiempo, es decir, la cantidad de distancia y tiempo para superar el obstáculo.

Obras buenas, hazlas a manos llenas; malas, ni una hagas. (= muchas)

\subsection{La expresión de la cantidad a través del trozo grande/pequeño de la vía}

Entre el prometer y el dar, largo trecho suele mediar. (= mucho)

De lo bonito a lo bueno, hay trecho. (= mucho)

Entre amar y aborrecer, poco trecho suele haber.(=poco)

Estos ejemplos representan la manifestación "espacial" de la cantidad en la metáfora popular y son bastante frecuentes.

\section{LA CANTIDAD EXPRESADA A TRAVÉS DE LA MEDIDA DE UN PESO O UN LÍQUIDO}

La cantidad puede venir expresada, como ya hemos dicho, a través de la medida de un peso o un líquido:

Entre risa y broma, se dicen verdades de a arroba. (= mucho) (la arroba - la medida del peso $=$ $11,5 \mathrm{~kg}$, y también la medida del líquido) 
Una onza de alegría vale más que mil doblillas. (= poco que vale mucho) En este caso el numeral mil no tiene el significado directo y sólo designa una gran cantidad.

Una onza de buen tinto vale más que una libra de talento.

Es de suponer que representando la cantidad a través de la medida concreta de un peso o de un líquido, el hispanohablante, probablemente, reproduce visualmente una u otra medida que se corresponde con cantidades grandes o pequeñas.

\section{PROVERBIOS DONDE LA CANTIDAD SE DETERMINA A TRAVÉS DE UN LAPSO DE TIEMPO GRANDE/PEQUEÑO}

En un dos por tres se vuelve el mundo al revés. (= en poco tiempo)

Ganancias del que juega no duran semana entera. (= no duran mucho)

Un mal paso a veces dura hasta la sepultura. (= mucho)

La mujer ángel es algún rato, y diablo, el resto del año. (= poco - mucho)

Lo que se hace a macha martillo dura siglos. (= mucho)

Ni lo dulce ni lo amargo dura tiempo largo. (= mucho)

En la boca de un gañán, en dos paletas se pierde un pan. (= en poco tiempo)

Dos amigos con una botella, en un santiamén dan cabo a ella. (= en poco tiempo)

Imaginación suelta, en un instante anda mil leguas. (= en poco tiempo anda mucho)

Se puede distinguir en el grupo especial proverbios en los que la cantidad se expresa a través de una unidad de tiempo expresada, a su vez, a través de la comparación con el objeto que tiene la característica espacial o con una parte del espacio:

Entre decir y hacer, una boyada suele caber. (= mucho)

En este caso, una boyada (monada de bueyes y vacas) ocupa un espacio grande, superarlo supondría un intervalo largo de tiempo.

Del desconsuelo al consuelo, no va un pelo. $(=$ poco $)$

Aquí el espacio ocupado iguala a un pelo que corresponde a un intervalo muy pequeño de tiempo.

De servil a ser vil va un clarito sutil. (= paco)

El período de tiempo para cambiar la conducta se expresa a través de un espacio muy estrecho.

Hablando de los proverbios arriba indicados, vemos que el tiempo, siendo una categoría significativa para la persona, nos permite expresar la idea de cualquier cantidad necesaria y cómo la consecuencia es el medio popular y frecuente para expresar la cantidad. 


\title{
5. LOS PROVERBIOS CON EL PRONOMBRE CUÁNTO EN LAS ORACIO- NES EXCLAMATIVAS
}

Hace falta destacar en un grupo aparte los proverbios con el pronombre cuánto en las oraciones exclamativas para transmitir la idea de cantidades grandes:

\author{
Una carita de pascua, ja cuántos engaña! (= a muchos) \\ La lumbre y el hecho, jcuántos pobres han hecho! (= muchos) \\ Fuiste doncella y viniste parida: ¡cuántas te tendrán envidia! (= muchas) \\ De una gota de un tintero, ¡cuánto malo y cuánto bueno! (= mucho) \\ Una gota de tinta, ¡cuánta honra puede dar y a cuántos la puede quitar! (= mucha/muchos)
}

\section{PROVERBIOS CON EL PRONOMBRE INTERROGATIVO QUÉ}

Los Proverbios con el pronombre interrogativo qué para expresar la ausencia de algo:

Donde no hay que comer, ¿qué alegría puede haber? (= no hay)

Del que no sabe ni la "Y", ¿qué se esperará? (= nada)

Los proverbios con cuánto expresan la idea de cantidades grandes, a diferencia de los proverbios con el componente qué, que indica la ausencia de algo. Las dos variantes son bastante frecuentes en la lengua española.

\section{LA PALABRA MONTÓN}

Por medio de la palabra montón en los rpoverbios se expresa una gran cantidad: Chispa pequeña enciende un montón de leña. (= mucho)

De lejanas regiones, mentiras a montones. (= muchas)

Años de nones, muchos montones. (= muchas)

El lexema montón contiene ya en su significado la idea de gran cantidad de algo, lo que explica su uso frecuente en los proverbios para expresar una cantidad grande.

\section{PROVERBIOS CON EL NUMERAL DOS}

Hay proverbios en los que se emplea el numeral dos con connotaciones negativas para expresar la idea de una cantidad muy pequeña:

Hombres sin peros no hubo dos; hubo uno era hombre y Dios. (= poco)

Hombre ni mujer sin pero, no hay dos en el mundo entero. (= poco)

El numeral dos es la primera contraposición a la singularidad, ya que no expresa una unidad y no expresa todavía una multitud de objetos, y la comparación con el numeral dos, especialmente con connotaciones negativas, da la posibilidad de expresar y subrayar una cantidad muy pequeña, mostrando casi su singularidad. 


\section{LOS PROVERBIOS QUE CONTIENEN LA CONTRAPOSICIÓN UNO/ CIENTO (CIENTO CON EL SIGNIFICADO «MUCHO»):}

De su suerte contentos, uno de cientos. (= pocos)

No hay santo como San Bruno, que da ciento por uno. (= mucho por poco)

El numeral ciento, además de su significado directo, tradicionalmente designaba una multitud de objetos, lo que explica su uso frecuente para expresar una cantidad grande y que sea habitual en la oposición uno/ciento(s).

\section{LOS PROVERBIOS, EN QUE EN LAS FIGURAS METAFÓRICAS ESTÁN PRESENTES LOS NÚMEROS DE UNO A DIEZ (LA PRIMERA DECENA):}

Hijos buenos-buenos, contados con los dedos. (= pocos)

Los numerales de la primera decena tienen un uso más frecuente en los proverbios, y casi todos son números-símbolo desde los tiempos antiguos.

Teniendo en cuenta lo arriba indicado, podemos afirmar que la noción de la cantidad en el proverbio español se expresa por medio de varias estructuras metafóricas, que tienen formas y estructuras léxicas diferentes. La cantidad, como una de las categorías básicas de la existencia, en el proverbio español se expresa a través de los conceptos básicos de la vida del pueblo: el tiempo, el espacio, la medida, la distancia y la forma.

En el presente artículo sólo tocamos el problema, el cual, según nuestro parecer, requiere un estudio más profundo, ya que la clasificación detallada y la descripción de los medios metafóricos que expresan la noción de la cantidad nos permitirá comprender el carácter tanto nacional como específico de la comprensión de las realidades por el pueblo español, cómo ve y qué piensa del mundo y de la gente.

\section{BIBLIOGRAFÍA}

Anikin, V.P. (1957): Los proverbios rusos, dichos, adivinanzas y el folclore infantil, Moscú, Uchpedguis.

Caudet, F. (1984): Los mejores refranes españoles, Madrid, Distribuciones Mateos.

Grishaeva, L.I. (2008): “Lengua como el código cultural o cómo los medios del idioma transmiten los conocimientos específicos culturales sobre el mundo?", Aspectos teoréticos y aplicados de la descripción de la lengua y la comunicación intercultural, Voronezh, Centro editorial de la Universidad Estatal de Voronezh, 222-250.

Lakoff, G. y Johnson, M.: “Metáforas de la vida cotidiana” http://www.durov.com/ linguistics1/lakoff-johnson-90.htm

Teliya, V. N. (1996): Fraseología rusa: los aspectos semántico, pragmático y linguoculturólogo, Moscú, Las lenguas de la cultura rusa. 